\title{
IS THERE AGREEMENT BETWEEN EVALUATORS THAT USED TWO SCORING SYSTEMS TO MEASURE ACUTE RADIATION DERMATITIS?
}

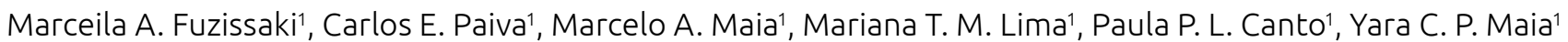

'Universidade Federal de Uberlância - Uberlândia (MG), Brazil.

Objective: To analyze the correlation between the Radiation Therapy Oncology Group (RTOG) and WHO (World Health Organization) scales and the agreement between evaluators. Methodology: A prospective and longitudinal study conducted during the period of 2016-2017, in a university hospital, including 834 images of irradiated sites of breast cancer (BC) women during radiotherapy (RT). The images were evaluated by three experienced nurses. In order to evaluate the correlation between the RTOG and WHO scales and the agreement between the evaluators, the Spearman correlation rho coefficient $(\rho)$ and the Kripp alpha ordinal agreement coefficient were used, respectively. The statistical software's used were SPSS version 20.0 and R version 3.2.5. Results: Moderate to strong correlations were identified between the two scales (correlation coefficients 0.4 - 0.9). When analyzing the agreement among the evaluators, moderate agreements (RTOG scale: 0.409 , 95\%CI $0.369-0.438$; WHO scale: 0.550 , 95\%CI 0.509-0.593) were identified and general agreement rates of $10.2 \%$ and $28.8 \%$, respectively, were obtained. Conclusions: The RTOG and WHO scales should be used with caution in the clinical practice to identify radiodermatitis prevalence and severity. An illustrative scale containing typical cases of radiodermatitis is being designed by our group aiming to improve accuracy and agreement between evaluators that will be tested in subsequent clinical studies. 